\title{
Prognostic Value of Late Gadolinium Enhancement on Cardiac Magnetic Resonance Imaging in Japanese Hypertrophic Cardiomyopathy Patients
}

\author{
Yasuki Hen, MD; Nobuo Iguchi, MD; Yuko Utanohara, MD; Kaori Takada, MD; \\ Haruhiko Machida, MD; Morimasa Takayama, MD; Tetsuya Sumiyoshi, MD
}

\begin{abstract}
Background: The prognostic value of late gadolinium enhancement (LGE) on contrast-enhanced cardiovascular magnetic resonance (CMR) in Japanese hypertrophic cardiomyopathy ( $\mathrm{HCM}$ ) patients in a large, single-center cohort was investigated.

Methods and Results: A total of $345 \mathrm{HCM}$ patients (mean age, 59 \pm 17 years; 214 male) underwent CMR with gadolinium enhancement, and were followed (mean duration, 21.8 months) for cardiovascular events. Patients were divided into event-positive and event-negative groups. The clinical and CMR characteristics were compared between the 2 groups, and predictors of cardiovascular events assessed on multivariate analysis. LGE was positive in 252 patients $(73 \%)$. The annual cardiovascular events rate was significantly higher in patients with LGE than in those without (6.2\%/year vs. 0.6\%/year, $\mathrm{P}=0.003$ ). On multivariate analysis, LGE (hazard ratio [HR], 7.436; 95\% confidence interval $[\mathrm{Cl}]$ : 1.001-55.228, $\mathrm{P}=0.050)$, increased myocardial mass index (HR, 1.013; 95\% Cl: 1.002-1.023, $\mathrm{P}=0.018)$, reduced left ventricular ejection fraction $(\mathrm{HR}, 0.965 ; 95 \% \mathrm{Cl}: 0.945-0.985, \mathrm{P}=0.001)$, and atrial fibrillation (HR, 2.257; 95\% Cl: 1.024-4.976, $\mathrm{P}=0.043$ ) were significantly associated with cardiovascular events.
\end{abstract}

Conclusions: The presence of LGE, increased myocardial mass index, reduced left ventricular ejection fraction and atrial fibrillation were independent predictors of adverse prognosis in Japanese HCM patients. (Circ J 2014; 78: 929-937)

Key Words: Cardiac magnetic resonance; Hypertrophic cardiomyopathy; Independent predictor; Late gadolinium enhancement

C ardiovascular events such as sudden death, admission for heart failure, heart failure death, and stroke are known complications of hypertrophic cardiomyopathy (HCM). ${ }^{1}$ Implantable cardioverter defibrillator (ICD) therapy has been reported to be effective, especially in preventing sudden death. ${ }^{2,3}$ Although multiple conventional risk factors associated with sudden death are not adequate for risk assessment of sudden death, ${ }^{4}$ they continue to be applied to clinical care. ${ }^{5}$

\section{Editorial p 832}

Recently, several studies of large cohorts have indicated that late gadolinium enhancement (LGE) observed on contrastenhanced cardiac magnetic resonance (CMR) imaging is a prognostic factor of sudden death and cardiovascular events, ${ }^{6-10}$ suggesting the potential clinical usefulness of this method. These studies, however, varied widely in the number of cases studied, follow-up period, definition of LGE, and endpoints. Hence, no conclusion has been reached so far. Furthermore, although there are several reports from Europe and the USA, no large-scale study has been reported from Japan. A definitive conclusion awaits further prospective long-term studies in multiple countries including Japan.

We conducted a retrospective study in a large number of HCM patients on the usefulness of LGE in predicting cardiovascular events.

\section{Methods}

\section{Patient Selection}

We conducted a retrospective study on $345 \mathrm{HCM}$ patients who underwent gadolinium contrast-enhanced CMR between 7 January 2006 and 31 December 2010. First, we explained to all the patients that their clinical data were to be used in a retrospec-

Received July 31, 2013; revised manuscript received December 8, 2013; accepted December 15, 2013; released online February 13, 2014 Time for primary review: 21 days

Department of Cardiology (Y.H., N.I., Y.U., M.T., T.S.), Department of Radiology (K.T.), Sakakibara Heart Institute, Tokyo; and Department of Radiology, Tokyo Women's Medical University Medical Center East, Tokyo (H.M.), Japan

Mailing address: Yasuki Hen, MD, Department of Cardiology, Sakakibara Heart Institute, 3-16-1 Asahi-cho, Fuchu, Tokyo 183-0003, Japan. E-mail: yhen@shi.heart.or.jp

ISSN-1346-9843 doi:10.1253/circj.CJ-13-0979

All rights are reserved to the Japanese Circulation Society. For permissions, please e-mail: cj@j-circ.or.jp 


\begin{tabular}{|c|c|c|c|c|}
\hline & All $(n=345)$ & Events (+) $(n=29)$ & Events $(-)(n=316)$ & P-value \\
\hline Age (years) & $59 \pm 17$ & $59 \pm 16$ & $58 \pm 19$ & 0.542 \\
\hline Male gender & $214(62.0)$ & $20(69.0)$ & $194(61.4)$ & 0.421 \\
\hline Family history of $\mathrm{HCM}$ & $39(11.3)$ & $3(10.3)$ & $36(11.4)$ & 1.000 \\
\hline Family history of SCD & $37(10.7)$ & $4(13.8)$ & $33(10.4)$ & 0.533 \\
\hline History of syncope & $44(12.8)$ & $4(13.8)$ & $40(12.7)$ & 0.775 \\
\hline DM & 46 (13.3) & $4(13.8)$ & $42(13.3)$ & 1.000 \\
\hline AF & $65(18.8)$ & $16(55.2)$ & $49(15.5)$ & 0.000 \\
\hline NYHA III or IV & $42(12.2)$ & $10(34.5)$ & $32(10.1)$ & 0.001 \\
\hline \multicolumn{5}{|l|}{ Medications } \\
\hline$\beta$-blocker & $236(68.4)$ & $23(79.3)$ & $213(67.4)$ & 0.187 \\
\hline Calcium antagonist & $97(28.1)$ & $7(24.1)$ & $90(28.5)$ & 0.619 \\
\hline
\end{tabular}

Data given as mean $\pm \mathrm{SD}$ or $\mathrm{n}(\%)$

AF, atrial fibrillation; CV, cardiovascular; DM, diabetes mellitus; HCM, hypertrophic cardiomyopathy; NYHA, New York Heart Association; SCD, sudden cardiac death

tive study, and then obtained patient consent in writing. This study was approved by the Ethics Committee of Sakakibara Heart Institute. HCM was defined as left ventricular (LV) wall thickness $\geq 15 \mathrm{~mm}$ on echocardiography and/or CMR, in the absence of other cardiac or systemic diseases that could account for the hypertrophy. Patients who were already diagnosed with HCM before undergoing CMR were recruited into this study even though their LV wall thickness was $<15 \mathrm{~mm}$. To investigate the prognosis of HCM, patients with old myocardial infarction, those who had concurrent congenital heart disease, those with severe valvular disease (including those who had undergone surgery for valvular diseases), and those who had undergone percutaneous transluminal septal myocardial ablation (PTSMA) or septal myectomy before CMR were excluded. Furthermore, we also excluded patients with renal dysfunction (estimated glomerular filtration rate $<30 \mathrm{ml} \cdot \mathrm{min}^{-1} \cdot 1.73 \mathrm{~m}^{-2}$ ).

\section{CMR Protocols and Image Analysis}

CMR was performed with a 1.5-T MR scanner (Magnetom Sonata; Siemens Medical Solutions, Erlangen, Germany) using a 6-channel phased-array body and spine coil. All images were acquired using the electrocardiography (ECG)-gated breathhold technique. First, steady-state free-precession cine images were acquired in 3 long-axis (2-, 3-, and 4-chamber) and 5 shortaxis views covering the $\mathrm{LV}$ from base to apex $(\mathrm{TR}=56.8 \mathrm{~ms}$, $\mathrm{TE}=1.2 \mathrm{~ms}$ ). Subsequently, LGE images were acquired $10 \mathrm{~min}$ after i.v. gadodiamide hydrate $0.1 \mathrm{mmol} / \mathrm{kg}$ (Omniscan; Daiichi Sankyo, Tokyo, Japan), using the inversion recovery technique in the identical views $(\mathrm{TR}=600 \mathrm{~ms}$, TE $=1.26 \mathrm{~ms}$; TI was individually optimized to null normal myocardial signal using a TI-scout sequence).

Experienced radiology technicians performed LV function analysis with dedicated software (Argus Function VA30; Siemens Medical Solutions). Epicardial and endocardial borders of the LV myocardium were manually traced during the whole cardiac phase on each cine short-axis image to obtain LV enddiastolic volume (EDV), end-systolic volume (ESV), ejection fraction (EF), and myocardial mass. The EDV index (EDVI) was calculated as EDV divided by the body surface area, and ESV index (ESVI), the ESV divided by the body surface area. Myocardial mass was calculated by multiplying the volume of the myocardium measured at end-diastole with the specific gravity of the myocardium $(1.05 \mathrm{~g} / \mathrm{ml})$. The $\mathrm{LV}$ mass index was calculated as myocardial mass divided by the body surface area of each patient.
Two cardiologists and 1 radiologist evaluated LGE images for the presence of LGE within the LV myocardium for each patient by consensus. The myocardial LGE areas were visually determined as those with higher signal intensity compared to the reference myocardium. The presence of any higher signal intensity area was defined as LGE positive. This spatial extension was assessed referring to the LV 17 -segment model. ${ }^{11}$ LGE was converted to scores on a scale of 0 to 17 , as the sum of the segments in the 17-segment model showing LGE. The maximum LV wall thickness was determined by measuring the minimal thickness of the thickest LV myocardium in the cine image at end-diastole.

\section{Clinical and CMR Status and Outcome}

The clinical records of all 345 patients were reviewed, and the clinical findings and medications at the time of CMR were extracted. Non-sustained ventricular tachycardia (NSVT) was not included in analysis because only 165 of 345 patients (48\%) underwent Holter ECG within 3 months of CMR. The same applied to low blood pressure during exercise, because the exercise test was performed in very few study patients. The CMR parameters analyzed were maximum LV wall thickness measured from cine-magnetic resonance imaging, as well as EDVI, ESVI, EF, and myocardial mass index calculated from LV functional analysis. In addition, the presence or absence of LGE was observed on gadolinium contrast-enhanced CMR.

In all 345 patients, the occurrence of cardiovascular events as of September 2011 was determined retrospectively from clinical records. Cardiovascular events were defined as cardiac death, sudden death, aborted sudden death, sustained ventricular tachycardia, appropriate ICD discharge, and unscheduled hospitalization for heart failure, stroke, or acute myocardial infarction. Cardiovascular events were subdivided into arrhythmic events defined as cardiac death due to incessant ventricular tachycardia/ ventricular fibrillation (VT/VF), sudden death, aborted sudden death, sustained VT, appropriate ICD discharge; and cardiac failure events defined as cardiac death due to progression of heart failure and unscheduled hospitalization for heart failure. The length of follow-up was until the most recent follow-up or death. For patients who underwent de novo PTSMA, surgical septal myectomy, or valve replacement during the follow-up period, the time at which these alternative endpoints occurred was regarded as the end of follow-up. During a mean follow-up of 21.8 \pm 19.2 months, PTSMA was performed in 71 patients $(21 \%)$, mitral valve replacement in 1 patient, and surgical sep- 


\begin{tabular}{|lccc|}
\hline Table 2. CMR Characteristics vs. Presence of CV Events & & \\
& Events $\mathbf{( + )}(\mathbf{n}=\mathbf{2 9})$ & Events $(\mathbf{-})(\mathbf{n}=\mathbf{3 1 6})$ & P-value \\
LVEDVI $\left(\mathrm{ml} / \mathrm{m}^{2}\right)$ & $74.1 \pm 21.6$ & $62.0 \pm 15.6$ & 0.006 \\
LVESVI $\left(\mathrm{ml} / \mathrm{m}^{2}\right)$ & $40.7 \pm 26.0$ & $20.9 \pm 10.9$ & 0.000 \\
EF $(\%)$ & $48.1 \pm 20.1$ & $67.1 \pm 10.1$ & 0.000 \\
LV myocardial mass index $\left(\mathrm{g} / \mathrm{m}^{2}\right)$ & $93.8 \pm 41.2$ & $71.4 \pm 27.9$ & 0.008 \\
Maximum LVWT $(\mathrm{mm})$ & $21.1 \pm 5.8$ & $20.6 \pm 5.0$ & 0.605 \\
Maximum LVWT $>30 \mathrm{~mm}$ & $3(10.3)$ & $24(7.6)$ & 0.486 \\
Presence of LGE & $28(96.6)$ & $224(70.9)$ & 0.003 \\
LGE score & $6.0 \pm 4.1$ & $3.1 \pm 3.0$ & 0.001 \\
\hline
\end{tabular}

Data given as mean $\pm \mathrm{SD}$ or $\mathrm{n}(\%)$.

CMR, cardiac magnetic resonance; EF, ejection fraction; LGE, late gadolinium enhancement; LV, left ventricular; LVEDVI, left ventricular end-diastolic volume index; LVESVI, left ventricular end-systolic volume index; LVWT, left ventricular wall thickness. Other abbreviation as in Table 1.

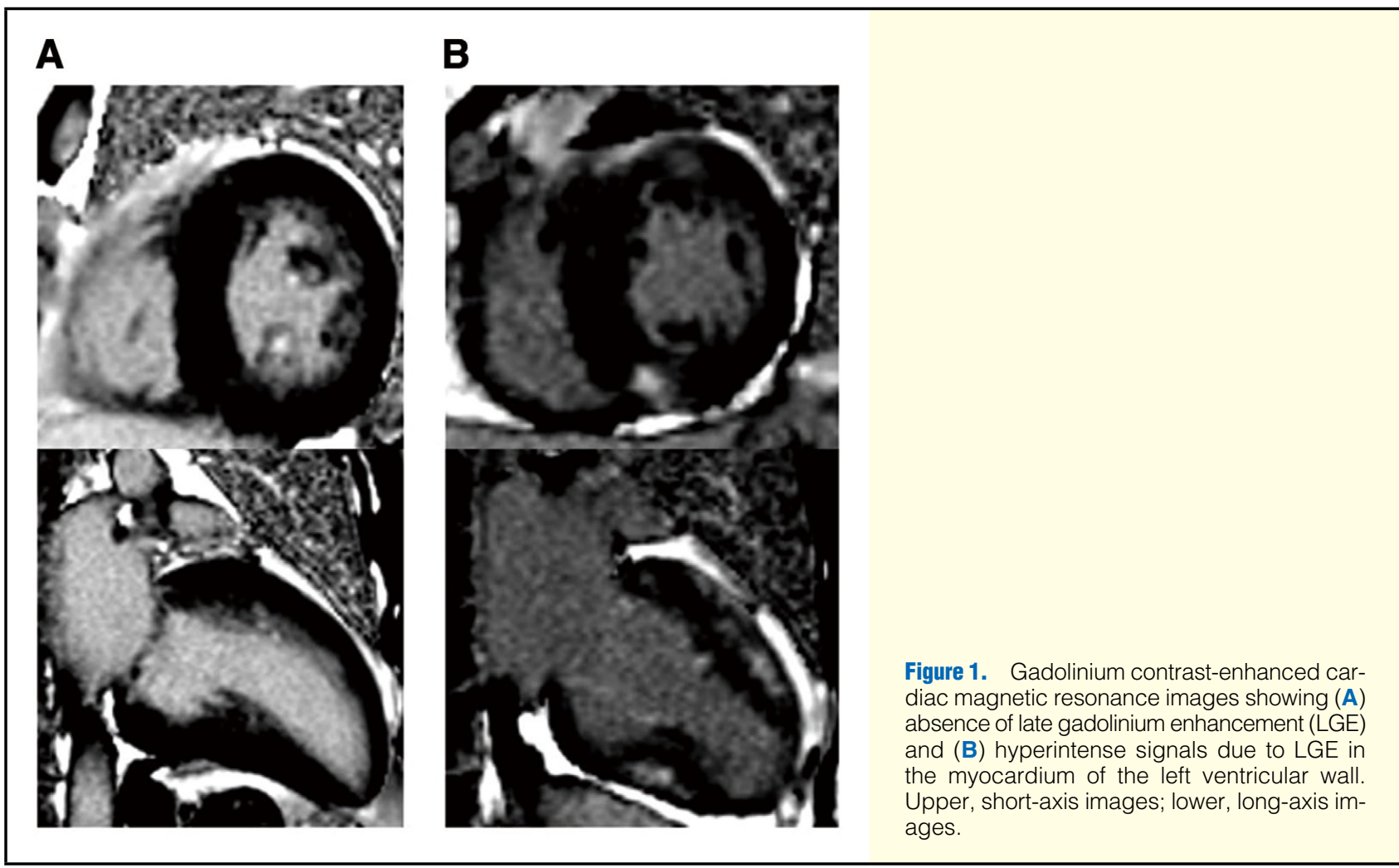

tal myectomy in 1 patient.

\section{Statistical Analysis}

Data are given as mean \pm SD for continuous variables and as frequency with percentage for categorical data. Differences between means were tested using unpaired Student's t-test. Frequencies were compared using chi-squared test or Fisher's exact test, as appropriate. Univariate analysis was performed using the clinical and CMR variables considered possibly related to cardiovascular events. Comparison of clinical and CMR characteristics in patients with or without LGE was done using logistic regression analysis. Furthermore, multivariate Cox regression analysis with stepwise variable selection was used to identify the predictors of cardiovascular events. $\mathrm{P} \leq 0.05$ was considered to be statistically significant. Analysis was performed using SPSS version 20.0.

\section{Results}

\section{Clinical and CMR Characteristics}

Table 1 lists the clinical characteristics of 345 patients divided into cardiovascular event-positive and -negative groups. Compared to the event-negative group, the event-positive group had a significantly higher rate of atrial fibrillation (AF; 16/29, 55.2\% vs. $49 / 316,15.5 \%, \mathrm{P}=0.000)$ and of New York Heart Association (NYHA) functional class III or IV (10/29, 34.5\% vs. 32/316, $10.1 \%, \mathrm{P}=0.001)$. The 2 groups did not differ significantly with respect to family history of sudden death or history of syncope, which are conventionally considered to be risk factors of sudden death.

Table 2 lists the CMR characteristics of 345 patients divided into cardiovascular event-positive and -negative groups. Compared to the event-negative group, the event-positive group had significantly larger EDVI (74.1 \pm 21.6 vs. $62.0 \pm 15.6 \mathrm{ml} / \mathrm{m}^{2}$, 


\begin{tabular}{|c|c|c|c|c|}
\hline & LGE (-) n =93 & LGE (+) n=252 & P-value & OR $(95 \% \mathrm{Cl})$ \\
\hline Age (years) & 65 & 57 & 0.000 & $0.964(0.947-0.982)$ \\
\hline Male gender & $45.2(42 / 93)$ & $68.3(172 / 252)$ & 0.000 & $2.611(1.604-4.249)$ \\
\hline Family history of $\mathrm{HCM}$ & $9.7(9 / 93)$ & $11.9(30 / 252)$ & 0.563 & $1.261(0.575-2.768)$ \\
\hline Family history of SCD & $6.5(6 / 93)$ & $12.3(31 / 252)$ & 0.126 & $2.034(0.820-5.046)$ \\
\hline History of syncope & $12.9(12 / 93)$ & $12.7(32 / 252)$ & 0.960 & $0.982(0.482-1.998)$ \\
\hline DM & $12.9(12 / 93)$ & $13.5(34 / 252)$ & 0.886 & $1.053(0.520-2.132)$ \\
\hline AF & $8.6(8 / 93)$ & $22.6(57 / 252)$ & 0.005 & $3.106(1.420-6.793)$ \\
\hline NYHA III or IV & $8.6(8 / 93)$ & $13.5(34 / 252)$ & 0.222 & $1.657(0.737-3.725)$ \\
\hline \multicolumn{5}{|l|}{ Medications } \\
\hline$\beta$-blocker & $72.0(67 / 93)$ & $67.1(169 / 252)$ & 0.378 & $0.790(0.468-1.334)$ \\
\hline Calcium antagonist & $25.8(24 / 93)$ & $29.0(73 / 252)$ & 0.562 & $1.172(0.684-2.009)$ \\
\hline \multicolumn{5}{|l|}{ CMR } \\
\hline LVEDVI (ml/m²) & 58.9 & 64.6 & 0.005 & $1.024(1.007-1.042)$ \\
\hline LVESVI (ml/m²) & 18.6 & 24.0 & 0.002 & $1.049(1.018-1.081)$ \\
\hline LVEF (\%) & 69.1 & 64.2 & 0.001 & $0.961(0.938-0.985)$ \\
\hline LV myocardial mass index $\left(\mathrm{g} / \mathrm{m}^{2}\right)$ & 59.8 & 78.3 & 0.000 & $1.029(1.017-1.041)$ \\
\hline Maximum LVWT (mm) & 17.3 & 21.9 & 0.000 & $1.319(1.216-1.430)$ \\
\hline
\end{tabular}

Data given as mean or as \% (n).

$\mathrm{Cl}$, confidence interval; LVEF, left ventricular ejection fraction; OR, odds ratio. Other abbreviations as in Tables 1,2.

\begin{tabular}{|c|c|c|c|c|c|c|c|}
\hline Patient & Events & $\begin{array}{c}\text { Age } \\
\text { (years) }\end{array}$ & Sex & Syncope & $\mathrm{FH}$ of SCD & $\mathrm{FH}$ of $\mathrm{HCM}$ & MWT $>30 \mathrm{~mm}$ \\
\hline 1 & HF hospitalization & 77 & Male & - & - & - & - \\
\hline 2 & Appropriate ICD discharge & 52 & Male & - & - & - & - \\
\hline 3 & HF hospitalization & 49 & Female & - & - & - & - \\
\hline 4 & Sudden death & 65 & Male & + & - & - & - \\
\hline 5 & HF hospitalization & 57 & Male & - & - & - & - \\
\hline 6 & HF hospitalization and appropriate ICD discharge & 66 & Male & - & - & - & - \\
\hline 7 & Appropriate ICD discharge & 59 & Male & - & - & - & - \\
\hline 8 & HF hospitalization & 56 & Female & - & - & + & - \\
\hline 9 & HF hospitalization & 45 & Male & - & - & - & - \\
\hline 10 & Stroke & 48 & Male & - & - & - & - \\
\hline 11 & HF hospitalization & 59 & Male & - & - & - & - \\
\hline 12 & HF hospitalization and incessant VT/VF & 80 & Female & - & - & - & - \\
\hline 13 & Acute myocardial infarction & 81 & Female & - & - & - & - \\
\hline 14 & HF hospitalization & 80 & Female & - & - & - & - \\
\hline 15 & HF hospitalization & 66 & Female & + & + & - & - \\
\hline 16 & Aborted sudden death & 18 & Female & - & + & - & - \\
\hline 17 & Appropriate ICD discharge & 76 & Male & + & + & - & - \\
\hline 18 & HF hospitalization & 71 & Male & - & - & - & - \\
\hline 19 & Aborted sudden death & 20 & Male & - & - & + & + \\
\hline 20 & Stroke & 57 & Male & - & - & - & - \\
\hline 21 & HF hospitalization & 41 & Male & - & + & + & - \\
\hline 22 & Appropriate ICD discharge & 13 & Female & + & - & - & - \\
\hline 23 & Appropriate ICD discharge & 39 & Male & - & - & - & + \\
\hline 24 & HF hospitalization & 74 & Female & - & - & - & - \\
\hline 25 & Stroke & 81 & Male & - & - & - & - \\
\hline 26 & HF hospitalization & 66 & Male & - & - & - & - \\
\hline 27 & Appropriate ICD discharge & 42 & Male & - & - & - & + \\
\hline 28 & HF hospitalization & 68 & Male & - & - & - & - \\
\hline 29 & Appropriate ICD discharge & 62 & Male & - & - & - & - \\
\hline
\end{tabular}


$\mathrm{P}=0.006)$, ESVI ( $40.7 \pm 26.0$ vs. $\left.20.9 \pm 10.9 \mathrm{ml} / \mathrm{m}^{2}, \mathrm{P}=0.000\right)$, and mass index $\left(93.8 \pm 41.2\right.$ vs. $\left.71.4 \pm 27.9 \mathrm{~g} / \mathrm{m}^{2}, \mathrm{P}=0.008\right)$. In contrast, EF was significantly smaller in the event-positive group compared to the event-negative group $(48.1 \pm 20.1$ vs. $67.1 \pm 10.1 \%$, $\mathrm{P}=0.000$ ). On contrast-enhanced CMR, LGE was observed in 252 of 345 patients $(73.0 \%)$. The prevalence of LGE was higher in the event-positive patients than in the event-negative patients $(28 / 29,96.6 \%$ vs. $224 / 316,70.9 \%, \mathrm{P}=0.003)$. LGE score was higher in the event-positive patients than in the eventnegative patients $(6.0 \pm 4.1$ vs. $3.1 \pm 3.0, \mathrm{P}=0.001)$. Figure 1 shows examples of the presence and absence of LGE in the LV myocardium.

Table 3 lists the clinical and CMR characteristics of 345 patients divided into LGE-positive and -negative groups. Compared to the LGE-negative group, the LGE-positive group had a significantly higher prevalence of male gender $(172 / 253,68.3 \%$ vs. $42 / 93,45.2 \%, \mathrm{P}=0.000)$ and $\mathrm{AF}(57 / 252,22.6 \%$ vs. $8 / 93$, $8.6 \%, \mathrm{P}=0.005)$. Compared to the LGE-negative group, the LGE-positive group was significantly younger ( 57 vs. 65 years old, $\mathrm{P}=0.000)$, and had significantly larger EDVI (64.6 vs. $\left.58.9 \mathrm{ml} / \mathrm{m}^{2}, \mathrm{P}=0.005\right)$, ESVI ( 24.0 vs. $\left.18.6 \mathrm{ml} / \mathrm{m}^{2}, \mathrm{P}=0.002\right)$, mass index ( 78.3 vs. $\left.59.8 \mathrm{~g} / \mathrm{m}^{2}, \mathrm{P}=0.000\right)$, and maximum $\mathrm{LV}$ wall thickness ( 21.9 vs. $17.3 \mathrm{~mm}, \mathrm{P}=0.000$ ). In contrast, $\mathrm{EF}$ was significantly smaller in the LGE-positive group compared to LGE-negative group (64.2 vs. $69.1 \%, \mathrm{P}=0.000)$.

\section{Follow-up Results}

During the follow-up period, cardiovascular events occurred in $28 / 252$ patients with LGE ( $11 \%$; sudden death in 1 , aborted sudden death in 2, appropriate ICD discharge in 7, stroke in 3 , acute myocardial infarction in 1 , and unscheduled hospitalization for heart failure in 14) and 1/93 patients without LGE (1\%; unscheduled hospitalization for heart failure). Table 4 shows the clinical and CMR imaging characteristics of 29 patients with cardiovascular events. Kaplan-Meier survival curves for cardiovascular events with regard to the presence of LGE are shown in Figure 2. Compared to patients without LGE, those with LGE had a higher rate of cardiovascular events (annual events rate, $6.2 \% /$ year vs. $0.6 \% / y e a r, \mathrm{P}=0.003$ ). Furthermore, outcome was compared with regard to the presence of LGE, focusing on arrhythmic events and heart failure events among cardiovascular events. One patient hospitalized for heart failure subsequently died due to incessant VT/VF. Another patient who was hospitalized had appropriate ICD discharge for VT during hospitalization. These 2 patients were analyzed and counted as having arrhythmic event. Figure 2 shows Kaplan-Meier survival curves for arrhythmic events and heart failure events with regard to the presence of LGE. Arrhythmic events occurred at a higher rate in patients with LGE than in those without LGE (annual events rate, $2.5 \% /$ year vs. $0 \%$ year, $\mathrm{P}=0.037$ ), while the rate of heart failure events was not significantly different between the

\begin{tabular}{|c|c|c|c|c|c|c|c|c|}
\hline Patient & NYHA & AF & $\begin{array}{l}\text { Mean LVEDVI } \\
\quad\left(\mathrm{ml}^{\mathrm{m}} \mathrm{m}^{2}\right)\end{array}$ & $\begin{array}{c}\text { Mean LVEF } \\
(\%)\end{array}$ & $\begin{array}{l}\text { Mean LVMI } \\
\left(\mathrm{g} / \mathrm{m}^{2}\right)\end{array}$ & $\begin{array}{l}\text { Mean MWT } \\
(\mathrm{mm})\end{array}$ & LGE & LGE score \\
\hline 1 & 4 & - & 35.0 & 47.5 & 140.5 & 25.0 & + & 4 \\
\hline 2 & 1 & - & 61.6 & 52.5 & 153.4 & 21.0 & + & 5 \\
\hline 3 & 3 & + & 107.4 & 25.8 & 102.5 & 16.0 & + & 6 \\
\hline 4 & 3 & - & 114.4 & 12.5 & 168.0 & 18.0 & + & 17 \\
\hline 5 & 3 & + & 83.2 & 20.8 & 81.2 & 9.0 & + & 4 \\
\hline 6 & 3 & + & 115.5 & 28.9 & 158.8 & 21.0 & + & 9 \\
\hline 7 & 2 & + & 46.7 & 62.0 & 87.2 & 26.0 & + & 9 \\
\hline 8 & 3 & - & 59.9 & 67.6 & 79.8 & 24.0 & + & 3 \\
\hline 9 & 3 & + & 68.4 & 39.0 & 90.8 & 24.5 & + & 6 \\
\hline 10 & 1 & + & 56.3 & 45.0 & 55.7 & 19.9 & + & 3 \\
\hline 11 & 1 & + & 78.1 & 18.0 & 112.6 & 14.4 & - & 0 \\
\hline 12 & 2 & + & 61.8 & 54.0 & 129.0 & 25.0 & + & 10 \\
\hline 13 & 1 & + & 63.8 & 77.0 & 88.9 & 18.9 & + & 2 \\
\hline 14 & 4 & - & 101.5 & 23.0 & 63.9 & 21.5 & + & 7 \\
\hline 15 & 2 & + & 62.6 & 55.0 & 57.7 & 19.1 & + & 4 \\
\hline 16 & 2 & - & 75.9 & 67.0 & 66.1 & 15.2 & + & 1 \\
\hline 17 & 1 & - & 55.9 & 53.2 & 67.9 & 21.3 & + & 4 \\
\hline 18 & 2 & + & 59.4 & 32.0 & 44.3 & 17.0 & + & 12 \\
\hline 19 & 2 & - & 92.8 & 43.0 & 177.2 & 32.0 & + & 7 \\
\hline 20 & 2 & - & 63.7 & 66.0 & 67.4 & 20.0 & + & 2 \\
\hline 21 & 3 & + & 114.0 & 21.8 & 58.7 & 14.0 & + & 6 \\
\hline 22 & 1 & - & 56.9 & 79.6 & 44.1 & 25.0 & + & 2 \\
\hline 23 & 2 & - & 82.1 & 49.3 & 155.5 & 32.0 & + & 11 \\
\hline 24 & 2 & + & 68.3 & 64.1 & 57.0 & 19.0 & + & 1 \\
\hline 25 & 1 & + & 87.0 & 73.4 & 81.0 & 22.0 & + & 4 \\
\hline 26 & 2 & + & 76.4 & 67.7 & 102.7 & 23.0 & + & 10 \\
\hline 27 & 1 & - & 51.5 & 66.0 & 131.8 & 35.0 & + & 8 \\
\hline 28 & 3 & + & 92.4 & 19.0 & 47.3 & 12.0 & + & 14 \\
\hline 29 & 1 & - & 55.6 & 63.0 & 47.9 & 22.0 & + & 4 \\
\hline
\end{tabular}

FH, family history; HF, heart failure; ICD, implantable cardioverter defibrillator; LVMI, left ventricular mass index; MWT, maximum wall thickness; VT/VF, ventricular tachycardia/ventricular fibrillation. Other abbreviations as in Tables 1,2,3. 
A

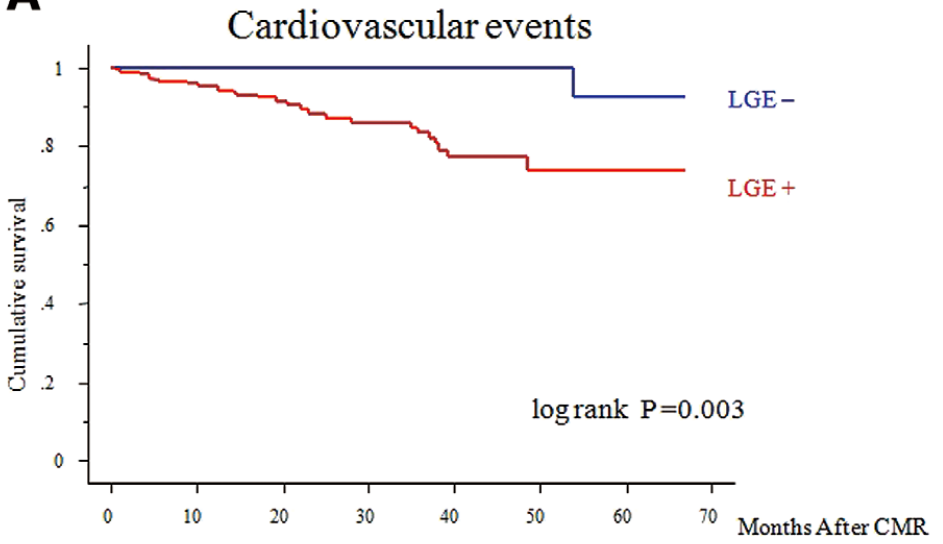

B

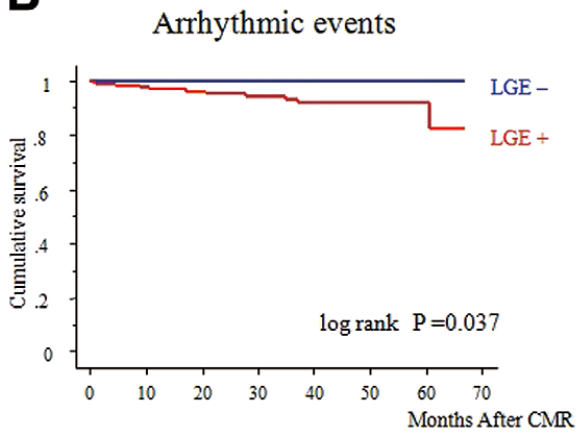

Heart failure events

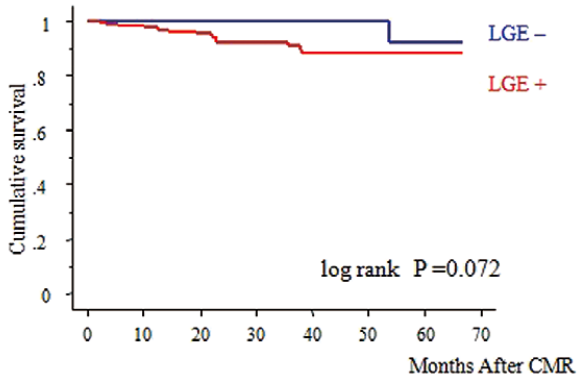

Figure 2. Kaplan-Meier curves of (A) cumulative cardiovascular, (B) arrhythmic and (C) heart failure event-free survival in late gadolinium enhancement (LGE)-positive and LGE-negative groups. Red, LGE positive; blue, LGE negative. CMR, cardiac magnetic resonance.

2 groups (annual events rate, 3.0\%/year vs. $0.6 \% /$ year, $\mathrm{P}=0.072$ ).

The patients were further divided into tertiles (no LGE, mild LGE, marked LGE) according to LGE score (first tertile, 0; second tertile, 1-3; third tertile, $>4)$. Optimal cut off score was determined on receiver operating characteristic (ROC) curve. Figure 3 shows Kaplan-Meier survival curves for cardiovascular events, arrhythmic events and heart failure events with regard to LGE score. The annual cardiovascular event rate in the marked LGE group exceeded that in the mild LGE and no LGE groups (8.4\%/year vs. $3.5 \%$ /year vs. $0.6 \%$ /year, $\mathrm{P}=0.001)$. The annual arrhythmic event rate in the marked LGE group exceeded that in the mild LGE and no LGE groups (3.7\%/year vs. 1.0\%/year vs. $0 \% / y e a r, \mathrm{P}=0.011)$. The annual heart failure event rate in the marked LGE group exceeded that in the mild LGE and no LGE groups (4.6\%/year vs. $1.0 \%$ /year vs. $0.6 \%$ /year, $\mathrm{P}=0.008$ ).

\section{Predictors of Cardiovascular Events}

When the clinical characteristics were compared between patients with and those without cardiovascular events, presence of AF and NHYA functional class III or IV were significantly different between the 2 groups. Analysis of CMR characteristics showed that EDVI, ESVI, EF, mass index, and the presence of LGE were significantly different between the 2 groups. Pairwise associations (EDVI, ESVI, EF, and mass index) were investigated using Pearson's correlation coefficient test. A significant $(\mathrm{P}<0.001)$ and strong $(\mathrm{r}=0.851)$ correlation was observed between EF and ESVI. As a result, ESVI was omitted while EF was selected for multivariate analysis. Finally, presence of AF; NYHA III or IV; EDVI; EF; mass index; and presence of LGE were included in the models, and multivariate Cox proportional hazards regression analysis was conducted after adjusting for age and sex. The results are listed in Table 5. On multivariate analysis, the presence of AF (hazard ratio [HR], 2.257; 95\% confidence interval [CI]: 1.024-4.976, $\mathrm{P}=0.043)$, reduced LVEF (HR, 0.965; 95\% CI: 0.945-0.985, $\mathrm{P}=0.001$ ), increased myocardial mass index (HR, 1.013; 95\% CI: 1.002-1.023, $\mathrm{P}=0.018$ ), and the presence of LGE (HR, 7.436; 95\% CI: 1.001-55.228, $\mathrm{P}=0.050$ ) were identified as independent prognostic factors of cardiovascular events.

\section{Discussion}

Although patients with HCM are generally regarded to have good prognosis, ${ }^{12}$ the occurrence of sudden death in some patients is an important issue. Moreover, other than sudden death, stroke death and heart failure death are also important causes of death in HCM patients. ${ }^{1}$

The conventional risk factors reported for sudden cardiac death in HCM include family history of sudden death, history of syncope, LV wall thickness $>30 \mathrm{~mm}$, NSVT, and low blood pressure during exercise. ${ }^{4}$ Prognosis is poorer as the number of risk factors increases, but it is a clinical fact that some patients who do not have these conventional risk factors may also die of sudden death. ${ }^{13,14}$ Maron et al studied HCM patients im- 
A

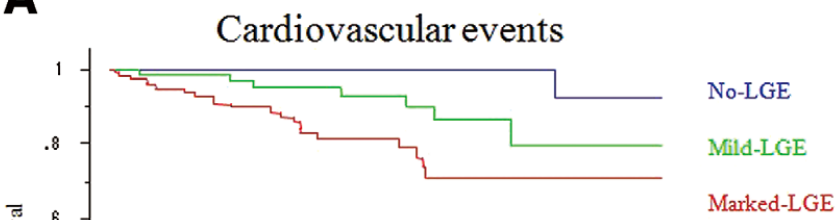

$\log$ rank $P=0.001$

B

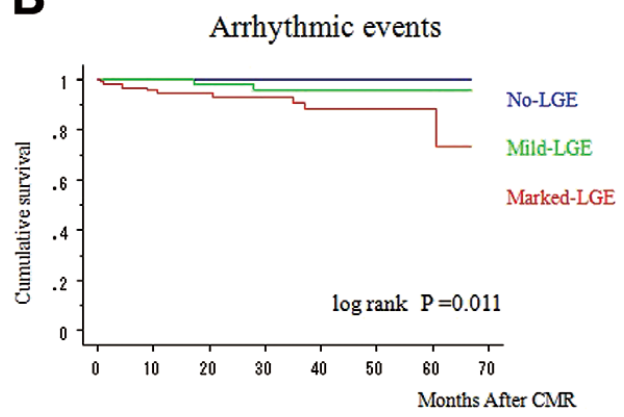

C

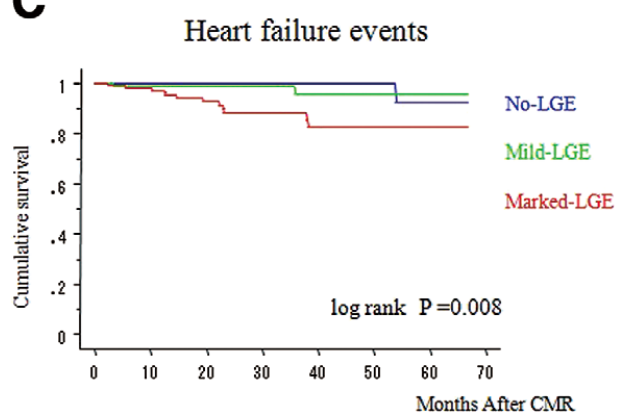

Figure 3. Kaplan-Meier curves of (A) cumulative cardiovascular, (B) arrhythmic and (C) heart failure event-free survival vs. extent of late gadolinium enhancement (LGE). Blue, no LGE (LGE score, 0); green, mild LGE (LGE score, 1-3); red, marked LGE (LGE score, $>4$ ). CMR, cardiac magnetic resonance

\begin{tabular}{|c|c|c|c|c|c|c|}
\hline \multirow{2}{*}{ Variable } & \multicolumn{3}{|c|}{ Univariate Analysis } & \multicolumn{3}{|c|}{ Multivariate Analysis } \\
\hline & Unadjusted HR & $(95 \% \mathrm{Cl})$ & P-value & Adjusted HR & $(95 \% \mathrm{Cl})$ & P-value \\
\hline Presence of AF & 4.166 & $2.002-8.67$ & 0.000 & 2.257 & $1.024-4.976$ & 0.043 \\
\hline NYHA III or IV & 5.953 & $2.760-12.838$ & 0.000 & & & \\
\hline LVEDVI $\left(\mathrm{ml} / \mathrm{m}^{2}\right)$ & 1.027 & $1.013-1.042$ & 0.000 & & & \\
\hline LVESVI (ml/m²) & 1.034 & $1.022-1.046$ & 0.000 & & & \\
\hline EF (\%) & 0.949 & $0.932-0.966$ & 0.000 & 0.965 & $0.945-0.985$ & 0.001 \\
\hline LV myocardial mass index $\left(\mathrm{g} / \mathrm{m}^{2}\right)$ & 1.020 & $1.010-1.030$ & 0.000 & 1.013 & $1.002-1.023$ & 0.018 \\
\hline Presence of LGE & 11.309 & $1.532-83.462$ & 0.017 & 7.436 & $1.001-55.228$ & 0.050 \\
\hline
\end{tabular}

$\mathrm{HR}$, hazard ratio. Other abbreviations as in Tables 1,2,3.

planted with ICD and observed no difference in the number of ICD discharges even when patients were stratified according to the number of risk factors. ${ }^{15}$ The existence of new prognostic factors other than the conventional risk factors is expected.

LGE on CMR has been reported as a unique method of evaluating myocardial damage. ${ }^{16,17}$ Many reports on the presence of LGE on CMR in HCM patients have been published since the first report in 2002. ${ }^{18}$ This LGE is presumed to represent mainly fibrosis of the myocardium, ${ }^{19,20}$ and the presence of LGE has been expected to be a prognostic factor of cardiovascular events, especially sudden death. In recent years, the relationship between the presence of LGE and prognosis has been investigated in relatively large-scale studies recruiting hundreds of patients. ${ }^{5-10}$
Maron et al suggested that there was no significant difference in the rate of combined cardiovascular events consisting of sudden death, appropriate ICD discharge, and progressive heart failure between patients with and those without LGE. ${ }^{6}$ Rubinshtein et al proposed that the presence of LGE may be a risk factor of sudden cardiac death, ${ }^{7}$ but the positive predictive value of LGE was low. Therefore, they commented that the absence of LGE does not mean that the risk is not present. Furthermore, O'Hanlon et al noted that the event rate was significantly higher in patients with fibrosis detected as LGE, and the risk of combined events increased with the increase in extent of fibrosis, ${ }^{8}$ but the extent of fibrosis was not a significant predictor for arrhythmic endpoint on multivariate analysis. Bruder et al reported that the presence of LGE was an independent prog- 
nostic factor of cardiac mortality, ${ }^{9}$ but sudden cardiac death was not significantly different on Kaplan-Meier survival curve analysis.

As noted here, although several studies have been reported, the endpoint as well as the evaluation method and definition of LGE differed among studies. Consequently no consensus opinion has been achieved. Currently a prospective long-term followup study enrolling a large number of patients using standardized LGE evaluation methods is ongoing. ${ }^{21}$

All the reports so far have been from Europe or the USA. The present study is the first conducted in Japan examining a large number of subjects. Multivariate analysis identified 4 factors as independent predictors of cardiovascular events: lowered $\mathrm{LVEF}$, increased myocardial mass index, presence of AF, and presence of LGE. This suggests that LGE is a prognostic factor of cardiovascular events in Japanese patients with HCM, as was reported in Western patients. A noteworthy finding in the present study is that in the group without LGE, there was only 1 case of unscheduled heart failure admission during follow-up and no other cardiovascular event.

$\mathrm{HCM}$ with decreased contractility is called end-stage HCM, ${ }^{22}$ and the prognosis of this is poor. ${ }^{23}$ Therefore, EF is an important prognostic factor for cardiovascular events in HCM, as in other types of cardiac disease. Compared to maximum LV wall thickness, myocardial mass has been reported to have a higher correlation with outcome, ${ }^{24}$ which would explain the relationship between abnormal increase in myocardial mass and event rate. In addition, $\mathrm{HCM}$ patients complicated with $\mathrm{AF}$ are known to have poorer outcome than those without AF. ${ }^{25,26}$ Thus, LVEF, myocardial mass index and AF are reasonable as independent risk factors of cardiovascular events. In addition to these 3 factors, the present study also identified the presence of LGE on contrast-enhanced CMR as an independent prognostic factor, but the P-value on multivariate analysis was 0.05 ; although this is considered significant, it cannot be interpreted as conclusive, as was the case in past studies. Several recent reports in a small number of patients, however, have indicated that the extent of LGE correlates with ventricular dysfunction and ventricular tachyarrhythmia. ${ }^{27-31} \mathrm{We}$ classified the present patients not only into 2 groups with regard to presence of LGE, but into 3 groups on LGE score; the prognosis was then seen to change gradually with LGE score. Based on the present results, gadolinium contrast-enhanced CMR can be recommended for evaluating prognosis in HCM if there are no contraindications.

\section{Study Limitations}

There were several limitations in this study. First, the study was a retrospective, single-center study conducted in a tertiary hospital, and the subjects did not include patients already implanted with ICD. Second, LGE evaluation was conducted visually, and was semi-quantitative. Third, given that PTSMA is actively performed in HCM patients, the mean follow-up period was short. Fourth, NSVT and low blood pressure during exercise test, which are conventional risk factors for sudden death, were not included in analyses because few patients underwent Holter ECG and/or exercise test at around the same time as CMR. To resolve these limitations, it would be necessary to carry out a prospective follow-up study recruiting a large number of patients with a protocol to perform CMR and Holter ECG at the time of diagnosis, and quantitative, standardized, LGE evaluation.

\section{Conclusions}

In 345 Japanese patients with HCM, LVEF, LV myocardial mass index, presence of AF, and presence of LGE were inde- pendent prognostic factors for cardiovascular events. In the absence of contraindications, CMR is recommended for prognosis evaluation in HCM patients.

\section{Acknowledgments}

This study was supported by the Sakakibara Clinical Research Grant for Promotion of Science, 2012. We gratefully thank Mr Naokazu Mizuno, Miss Airi Shibata, and Mr Shou Sugioka for their technical assistance.

\section{References}

1. Maron BJ, Olivotto I, Spirito P, Casey SA, Bellone P, Gohman TE, et al. Epidemiology of hypertrophic cardiomyopathy-related death: Revisited in a large non-referral-based patient population. Circulation 2000; 102: 858-864.

2. Maron BJ, Shen WK, Link MS, Epstein AE, Almquist AK, Daubert JP, et al. Efficacy of implantable cardioverter-defibrillators for the prevention of sudden death in patients with hypertrophic cardiomyopathy. $N$ Engl J Med 2000; 342: 365-373.

3. Jayatilleke I, Doolan A, Ingles J, McGuire M, Booth V, Richmond DR, et al. Long-term follow-up of implantable cardioverter defibrillator therapy for hypertrophic cardiomyopathy. Am J Cardiol 2004; 93: $1192-1194$.

4. Elliott PM, Poloniecki J, Dickie S, Sharma S, Monserrat L, Varnava A, et al. Sudden death in hypertrophic cardiomyopathy: Identification of high risk patients. J Am Coll Cardiol 2000; 36: 2212-2218.

5. Maron BJ. Risk stratification and role of implantable defibrillators for prevention of sudden death in patients with hypertrophic cardiomyopathy. Circ J 2010; 74: 2271-2282.

6. Maron MS, Appelbaum E, Harrigan CJ, Buros J, Gibson CM, Hanna $\mathrm{C}$, et al. Clinical profile and significance of delayed enhancement in hypertrophic cardiomyopathy. Circ Heart Fail 2008; 1: 184-191.

7. Rubinshtein R, Glockner JF, Ommen SR, Araoz PA, Ackerman MJ, Sorajja $\mathrm{P}$, et al. Characteristics and clinical significance of late gadolinium enhancement by contrast-enhanced magnetic resonance imaging in patients with hypertrophic cardiomyopathy. Circ Heart Fail 2010; 3: $51-58$.

8. O'Hanlon R, Grasso A, Roughton M, Moon JC, Clark S, Wage R, et al. Prognostic significance of myocardial fibrosis in hypertrophic cardiomyopathy. J Am Coll Cardiol 2010; 56: 867-874.

9. Bruder O, Wagner A, Jensen CJ, Schneider S, Ong P, Kispert EM, et al. Myocardial scar visualized by cardiovascular magnetic resonance imaging predicts major adverse events in patients with hypertrophic cardiomyopathy. J Am Coll Cardiol 2010; 56: 875-887.

10. Green JJ, Berger JS, Kramer CM, Salerno M. Prognostic value of late gadolinium enhancement in clinical outcomes for hypertrophic cardiomyopathy. JACC Cardiovasc Imaging 2012; 5: 370-377.

11. Cerqueira MD, Weissman NJ, Dilsizian V, Jacobs AK, Kaul S, Laskey WK, et al. Standardized myocardial segmentation and nomenclature for tomographic imaging of the heart: A statement for healthcare professionals from the Cardiac Imaging Committee of the Council on Clinical Cardiology of the American Heart Association. Circulation 2002; 105: 539-542.

12. Maron BJ, Casey SA, Poliac LC, Gohman TE, Almquist AK, Aeppli DM. Clinical course of hypertrophic cardiomyopathy in a regional United States cohort. JAMA 1999; 281: 650-655.

13. Maron BJ, Maron MS, Lesser JR, Hauser RG, Haas TS, Harrigan CJ, et al. Sudden cardiac arrest in hypertrophic cardiomyopathy in the absence of conventional criteria for high risk status. Am J Cardiol 2008; 101: $544-547$.

14. Bongioanni S, Spirito P, Masi AS, Chiribiri A, Bonamini R, Conte MR. Extensive myocardial fibrosis in a patient with hypertrophic cardiomyopathy and ventricular tachycardia without traditional highrisk features. Circ Cardiovasc Imaging 2009; 2: 349-350.

15. Maron BJ, Spirito P, Shen WK, Haas TS, Formisano F, Link MS, et al. Implantable cardioverter-defibrillators and prevention of sudden cardiac death in hypertrophic cardiomyopathy. JAMA 2007; 298: 405412.

16. Nakamori S, Matsuoka K, Onishi K, Kurita T, Ichikawa Y, Nakajima $\mathrm{H}$, et al. Prevalence and signal characteristics of late gadolinium enhancement on contrast-enhanced magnetic resonance imaging in patients with takotsubo cardiomyopathy. Circ J 2012; 76: 914-921.

17. Sato T, Tsujino I, Ohira H, Oyama-Manabe N, Yamada Y, Otsuka N, et al. Right atrial late gadolinium enhancement on cardiac magnetic resonance imaging in pulmonary hypertension. Circ J 2012; 76: $238-$ 239.

18. Choudhury L, Mahrholdt H, Wagner A, Choi KM, Elliott MD, Klocke FJ, et al. Myocardial scarring in asymptomatic or mildly symptomatic patients with hypertrophic cardiomyopathy. J Am Coll Cardiol 2002; 
40: $2156-2164$.

19. Moon JC, Reed E, Sheppard MN, Elkington AG, Ho SY, Burke M, et al. The histologic basis of late gadolinium enhancement cardiovascular magnetic resonance in hypertrophic cardiomyopathy. $\mathrm{J} \mathrm{Am} \mathrm{Coll}$ Cardiol 2004; 43: 2260-2264.

20. Papavassiliu T, Schnabel P, Schroder M, Borggrefe M. CMR scarring in a patient with hypertrophic cardiomyopathy correlates well with histological findings of fibrosis. Eur Heart J 2005; 26: 2395.

21. Wagner A, Bruder O, Schneider S, Nothnagel D, Buser P, Pons-Lado $\mathrm{G}$, et al. Current variables, definitions and endpoints of the European cardiovascular magnetic resonance registry. J Cardiovasc Magn Reson 2009; 11: 43

22. Harris KM, Spirito P, Maron MS, Zenovich AG, Formisano F, Lesser $\mathrm{JR}$, et al. Prevalence, clinical profile, and significance of left ventricular remodeling in the end-stage phase of hypertrophic cardiomyopathy. Circulation 2006; 114: 216-225.

23. Biagini E, Coccolo F, Ferlito M, Perugini E, Rocchi G, Bacchi-Reggiani L, et al. Dilated-hypokinetic evolution of hypertrophic cardiomyopathy: Prevalence, incidence, risk factors, and prognostic implications in pediatric and adult patients. J Am Coll Cardiol 2005; 46: 1543-1550.

24. Olivotto I, Maron MS, Autore C, Lesser JR, Rega L, Casolo G, et al. Assessment and significance of left ventricular mass by cardiovascular magnetic resonance in hypertrophic cardiomyopathy. J Am Coll Cardiol 2008; 52: 559-566.

25. Olivotto I, Cecchi F, Casey SA, Dolara A, Traverse JH, Maron BJ. Impact of atrial fibrillation on the clinical course of hypertrophic cardiomyopathy. Circulation 2001; 104: 2517-2524.
26. Kubo T, Kitaoka H, Okawa M, Hirota T, Hayato K, Yamasaki N, et al. Clinical impact of atrial fibrillation in patients with hypertrophic cardiomyopathy. Results from Kochi RYOMA Study. Circ J 2009; 73: $1599-1605$.

27. Moon J, Hong YJ, Kim YJ, Shim CY, Jang Y, Chung N, et al. Extent of late gadolinium enhancement on cardiovascular magnetic resonance imaging and its relation to left ventricular longitudinal functional reserve during exercise in patients with hypertrophic cardiomyopathy. Circ J 2013; 77: 1742 - 1749 .

28. Fluechter S, Kuschyk J, Wolpert C, Doesch C, Veltmann C, Haghi $\mathrm{D}$, et al. Extent of late gadolinium enhancement detected by cardiovascular magnetic resonance correlates with the inducibility of ventricular tachyarrhythmia in hypertrophic cardiomyopathy. J Cardiovasc Magn Reson 2010; 12: 30.

29. Prinz C, Schwarz M, Ilic I, Laser KT, Lehmann R, Prinz EM, et al. Myocardial fibrosis severity on cardiac magnetic resonance imaging predicts sustained arrhythmic events in hypertrophic cardiomyopathy. Can J Cardiol 2013; 29: 358-363.

30. Kwon DH, Setser RM, Popovic ZB, Thamilarasan M, Sola S, Schoenhagen $\mathrm{P}$, et al. Association of myocardial fibrosis, electrocardiography and ventricular tachyarrhythmia in hypertrophic cardiomyopathy: A delayed contrast enhanced MRI study. Int J Cardiovasc Imaging 2008; 24: 617-625.

31. Leonardi S, Raineri C, De Ferrari GM, Ghio S, Scelsi L, Pasotti M, et al. Usefulness of cardiac magnetic resonance in assessing the risk of ventricular arrhythmias and sudden death in patients with hypertrophic cardiomyopathy. Eur Heart J 2009; 30: 2003-2010. 